\title{
A fuzzy majority-based construction method for composed aggregation functions by using combination operator
}

\begin{abstract}
This paper focuses on the problem of constructing aggregation functions which support the concept of partial agreement when only one or more sub-group(s) of decision makers and criteria, not necessarily all of them, is (are) involved to take the final action. The proposed method is started with computing all different $\alpha$-combinations from an n-element set of inputs, according to the binomial coefficient, and then completed by combining their aggregations. This approach, so-called combination operator-based aggregation function, guarantees to consider various agreement scenarios at different consensus levels in contrary with the traditional composition-based construction methods that act as a full agreement. The attention is then given to the weighted case specially the conditions of weighting vectors for ensuring the well-defined property of the proposed method. As an application, the combination operatorbased aggregation function is generalized over the Cartesian product of unit intervals $[0,1]$ to deal with multi-polar information. The proposed aggregating methodology is then used to reach consensus in a group decision-making problem.
\end{abstract}

Keyword: Aggregation function; Combination operator; Weighting matrix; Decision-making; Consensus 